\title{
Stationary solutions and spatial-temporal dynamics of a shadow system of LV competition models
}

Yuanyuan Zhang ${ }^{1}$ and Li Xia $2,3^{*}$

\section{"Correspondence:}

xaleysherry@163.com

${ }^{2}$ College of Mathematics and

Statistics, Guangdong University of

Finance and Economics,

Guangzhou, Guangdong 510320,

China

${ }^{3}$ College of Mathematics and

Statistics, Shenzhen University,

Shenzhen, Guangdong 518060

China

Full list of author information is

available at the end of the article

\begin{abstract}
The concern of the paper is nonconstant positive solutions of a class of Lotka-Volterra competition systems over 1D domains. We prove the existence of a positive monotonous solution to the shadow system for each small diffusion rate $\epsilon>0$. Our theoretical results provide a foundation for further theoretical analysis on the shadow system and give insights on how diffusion and advection rates affect the pattern formation in the advective Lotka-Volterra competition systems. The second part of this paper includes numerical simulations of the nontrivial patterns to the shadow system and its original model. It is demonstrated that nontrivial patterns can develop from small perturbations of the homogeneous solution. Our numerics suggest that this system admits very interesting and complicated spatial-temporal dynamics even over 1D domains.
\end{abstract}

Keywords: steady state; spatial-temporal dynamics; Lotka-Volterra competition system; shadow system

\section{Introduction}

This paper is concerned with the following boundary value problem with integral constraint:

$$
\left\{\begin{array}{l}
\epsilon v_{\epsilon}^{\prime \prime}+\left(a_{2}-b_{2} \lambda_{\epsilon} e^{-r \Phi\left(v_{\epsilon}\right)}-c_{2} v_{\epsilon}\right) v_{\epsilon}=0, \quad x \in(0, L), \\
\int_{0}^{L}\left(a_{1}-b_{1} \lambda_{\epsilon} e^{-r \Phi\left(v_{\epsilon}\right)}-c_{1} v_{\epsilon}\right) e^{-r \Phi\left(v_{\epsilon}\right)} d x=0, \\
v_{\epsilon}(x)>0, x \in(0, L) ; \quad v_{\epsilon}^{\prime}(0)=v_{\epsilon}^{\prime}(L)=0,
\end{array}\right.
$$

where $\epsilon$ is a positive constant, $v_{\epsilon}$ is a function of $x$, and $\lambda_{\epsilon}$ is an unknown constant. Parameters $a_{i}, b_{i}, c_{i}, i=1,2$, and $r$ are positive constants, and $\Phi$ is a smooth function. System (1.1) is the 1D shadow system to the following model:

$$
\begin{cases}\nabla \cdot\left(d_{1} \nabla u+\chi u \phi(v) \nabla v\right)+\left(a_{1}-b_{1} u-c_{1} v\right) u=0, & x \in \Omega, \\ d_{2} \Delta v+\left(a_{2}-b_{2} u-c_{2} v\right) v=0, & x \in \Omega, \\ \partial_{\nu} u=\partial_{\nu} v=0, & x \in \partial \Omega,\end{cases}
$$

(c) The Author(s) 2017. This article is distributed under the terms of the Creative Commons Attribution 4.0 International License (http://creativecommons.org/licenses/by/4.0/), which permits unrestricted use, distribution, and reproduction in any medium, provided you give appropriate credit to the original author(s) and the source, provide a link to the Creative Commons license, and indicate if changes were made. 
which was proposed in [1] to study the aggregation phenomenon of two competing species subject to Lotka-Volterra kinetics. Here $\Omega \subset \mathbb{R}^{N}, N \geq 1$, is a bounded domain with piecewise smooth boundary $\partial \Omega$, and $v$ is the unit outer normal on the boundary. See [1] for the derivation of this model and biological justifications for the system parameters.

System (1.1) serves as the shadow system of (1.2) on the asymptotic behaviors of $(u, v)$ in the limit of large advection rate $\chi$, and it admits spatial structures such as spikes, transition layers, and so on. To demonstrate our motivation for the study of (1.1), we first recall the following results on (1.2) established in [1] for $\Omega=(0, L)$. Let $\left(u_{i}, v_{i}\right)$ be positive solutions of (1.2) with $\left(d_{1, i}, d_{2, i}, \chi_{i}\right)=\left(d_{1}, d_{2}, \chi\right)$. Suppose that $\chi_{i} \rightarrow \infty, d_{2, i} \rightarrow d_{2} \in(0, \infty)$, and $\frac{\chi_{i}}{d_{1, i}}=$ $r_{i} \rightarrow r \in(0, \infty)$ as $i \rightarrow \infty$. It is proved in Theorem 4.2 in [1] that there exists a nonnegative constant $\lambda_{\infty}$ such that

$$
u_{i} e^{r_{i} \Phi\left(v_{i}\right)} \rightarrow \lambda_{\infty} \quad \text { uniformly on }[0, L]
$$

where

$$
\Phi(s)=\int_{0}^{s} \phi(r) d r
$$

moreover, after passing to a subsequence if necessary, as $i \rightarrow \infty$,

$$
\left(u_{i}, v_{i}\right) \rightarrow\left(\lambda_{\infty} e^{-r \Phi\left(v_{\infty}\right)}, v_{\infty}\right) \quad \text { in } C^{1}([0, L]) \times C^{1}([0, L])
$$

where $v_{\infty}=v_{\infty}(x)$ satisfies

$$
\left\{\begin{array}{l}
d_{2} v_{\infty}^{\prime \prime}+\left(a_{2}-b_{2} \lambda_{\infty} e^{-r \Phi\left(v_{\infty}\right)}-c_{2} v_{\infty}\right) v_{\infty}=0, \quad x \in(0, L) \\
\int_{0}^{L}\left(a_{1}-b_{1} \lambda_{\infty} e^{-r \Phi\left(v_{\infty}\right)}-c_{1} v_{\infty}\right) e^{-r \Phi\left(v_{\infty}\right)} d x=0 \\
v_{\infty}^{\prime}(0)=v_{\infty}^{\prime}(L)=0 .
\end{array}\right.
$$

Here $v_{\infty}(x)$ is a nonnegative function of $x$. Since small diffusion rate $d_{2}$ supports a nontrivial solution of (1.3), we denote $d_{2}=\epsilon$ and put $\left(v_{\infty}, \lambda_{\infty}\right)=\left(v_{\epsilon}, \lambda_{\epsilon}\right)$, and then (1.3) becomes (1.1).

In this paper, we investigate the existence of a nonconstant positive solution to system (1.1). To this end, we analyze the global bifurcation properties of system (1.1) and show that the global continuum of the first branch must be noncompact in certain Banach spaces. In particular, we prove that, for each small $\epsilon>0,(1.1)$ always admits a nonconstant positive solution $v_{\epsilon}(x)$, which is monotone in $(0, L)$; see Theorem 1.1. The global bifurcation is important especially when nonconstant positive solutions are concerned, and our results provide a foundation for further analysis on the shadow system (1.1), compared to the local branches, which have been investigated in detail in [1].

From the view point of mathematical modeling, a fundamental problem in mathematical ecology is to study the spatial-temporal evolutions of mutually interacting species. In particular, one of the most interesting phenomena is the well-observed segregation of species through interspecific competition. It is well known that the classical diffusive Lotka-Volterra competition system (i.e., (1.2) with $\chi=0$ ) only admits constant stable steady states except when $\Omega$ is nonconvex and the diffusion rates $d_{i}$ are large [2-7]. We refer to [1] for further discussions. In order to take into account population pressures due 
to the presence of interacting species, various cross-diffusion models have been proposed and extensively studied over the past few decades. One celebrated system was proposed by Shigesada, Kawasaki, and Teramoto [8] in 1979, now often referred to as the SKT model, and it can be used to describe the aforementioned segregation phenomena. We refer to [9-11] for works on the SKT competition model.

It is necessary to point out that the process of cross-diffusion is very similar to that of chemotaxis, in which cellular organisms direct their dispersals toward or against the concentration gradient of stimulating chemicals in the environment. The mathematical modeling of chemotaxis dates back to the pioneering works of Keller and Segel [12]. Very recently, in [1] and [13], there was independently proposed and studied the Keller-Segel type chemotaxis model (1.1), for which it is assumed that species $u$ takes a combination of random and directed dispersal strategy, whereas species $v$ only disperses randomly. In particular, both papers investigated the existence of nontrivial solutions to (1.2) through its shadow system, and transition layer and boundary spike solutions to (1.1) have been established. These nontrivial positive solutions to (1.1) describe the coexistence and segregation of the competing species in the limit of large diffusion rate $d_{1}$ and advection rate $\chi$.

To illustrate how nonconstant positive solutions to (1.1) are established in [1] and provide necessary settings for our coming analysis, we first note that (1.1) has a unique positive constant solution

$$
(\bar{v}, \bar{\lambda})=\left(\frac{a_{2} b_{1}-a_{1} b_{2}}{b_{1} c_{2}-b_{2} c_{1}}, \frac{a_{2} b_{1}-a_{1} b_{2}}{b_{1} c_{2}-b_{2} c_{1}} e^{-r \Phi(\bar{v})}\right),
$$

provided that

$$
\frac{c_{1}}{c_{2}}<\frac{a_{1}}{a_{2}}<\frac{b_{1}}{b_{2}} \quad \text { or } \quad \frac{b_{1}}{b_{2}}<\frac{a_{1}}{a_{2}}<\frac{c_{1}}{c_{2}} .
$$

In [1], Crandall-Rabinowitz bifurcation theory [14] is applied to establish the existence of nonconstant positive solutions to (1.1) bifurcating from $(\bar{v}, \bar{\lambda})$. To be precise, let

$$
\epsilon_{n}=\frac{\left(\left(a_{2}-c_{2} \bar{v}\right) r \Phi^{\prime}(\bar{v})-c_{2}\right) \bar{v}}{(n \pi / L)^{2}}>0, \quad n \in \mathbb{N}^{+}
$$

It is proved in Theorem 4.3 of [1] that the steady-state bifurcation occurs at $\left(\bar{v}, \bar{\lambda}, \epsilon_{n}\right)$ for each $n \in \mathbb{N}^{+}$, with

$$
\begin{aligned}
& \epsilon_{n}(s)=\epsilon_{n}+\mathcal{K} s^{2}+O\left(s^{3}\right), \\
& \left(v_{n}(x, s), \lambda_{n}(s)\right)=(\bar{v}, \bar{\lambda})+s\left(\cos \frac{n \pi x}{L}, 0\right)+O\left(s^{2}\right),
\end{aligned}
$$

and there exist a constant $\delta>0$ and continuous functions $\left(v_{n}(x, s), \lambda_{n}(s), \epsilon_{n}(s)\right): s \in$ $(-\delta, \delta) \rightarrow \mathcal{X} \times \mathbb{R}^{+} \times \mathbb{R}^{+}$such that, for each $s \in(-\delta, \delta),\left(v_{n}(x, s), \lambda_{n}(s), \epsilon_{n}(s)\right)$ solves system (1.1), where

$$
\mathcal{X}=\left\{w \in H^{2}(0, L) \mid w^{\prime}(0)=w^{\prime}(L)=0\right\}
$$


moreover, all nontrivial solutions $(v, \lambda, \epsilon)$ of $(1.1)$ near $\left(\bar{v}, \bar{\lambda}, \epsilon_{n}\right)$ must be on the $n$th bifurcation branch

$$
\Gamma_{n}(s):=\left\{\left(v_{n}(x, s), \lambda_{n}(s), \epsilon_{n}(s)\right): s \in(-\delta, \delta)\right\} .
$$

We would like to mention that the stability or instability of these bifurcating solutions (1.6) are also investigated in [1], Theorem 4.4. They showed that, among all the local bifurcating solutions, only those on the first branch $\Gamma_{1}(s), s \in(-\delta, \delta)$, can be stable, provided that $\mathcal{K}>0$, whereas all the remaining local branches are unstable. This corresponds to [15], where it is stated that the nonconstant stable solutions to a classical of shadow systems must be spatially monotone. These results make the current study of the first bifurcation branch more realistic for further applications.

One of the motivations of this paper is to study the topological behaviors of the local branches $\Gamma_{n}(s)$ to (1.1). To this end, we perform the global bifurcation analysis for (1.1); moreover, we shall show that this shadow system always admits nonconstant positive solutions for each small $\epsilon>0$. To be precise, the theoretical result of the paper states as follows.

Theorem 1.1 Assume that (1.4) is satisfied and $\epsilon_{n}$ is given by (1.5). Then, for each $\epsilon \in\left(0, \epsilon_{1}\right)$, there exists a positive solution $\left(v_{\epsilon}(x), \lambda_{\epsilon}\right)$ of $(1.1)$ satisfying $v_{\epsilon}^{\prime}<0$ on $(0, L)$.

We present the plots in Figure 1 to illustrate the first bifurcation branch or one of the solution sets to (1.1) schematically. Each element except $\left(\bar{v}, \bar{\lambda}, \epsilon_{1}\right)$ on the bifurcation branch represents a nontrivial solutions to (1.1). Our analysis shows that the project of each branch onto the $\epsilon$-axis must include the interval $\left(0, \epsilon_{1}\right)$.

Remark 1 Theorem 1.1 indicates that (1.1) admits decreasing solutions for every small $\epsilon$ and the proof can be carried over to show that this system also admits increasing solutions. Indeed, let $v_{\epsilon}(x)$ be a decreasing solution of (1.1). Then, thanks to the Neumann boundary condition, $v_{\epsilon}(L-x)$ is also a solution, and it is increasing in $x$. Therefore, we can construct

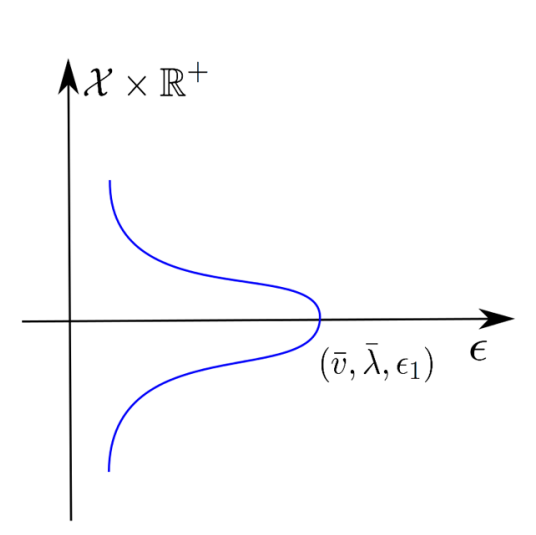

Subcritical bifurcation branch $\Gamma_{1}(s)$

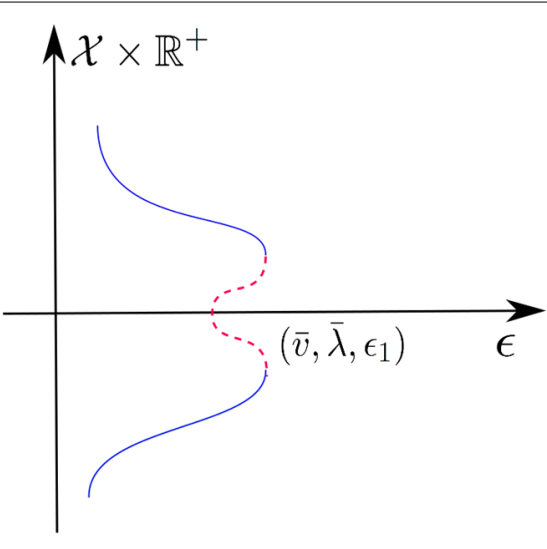

Supercritical bifurcation branch $\Gamma_{1}(s)$

Figure 1 Pitchfork-type bifurcation branches. The solid line represents stable bifurcating solution $\left(v_{1}(s, x), \lambda_{1}(s), \epsilon_{1}(s)\right)$, and the dashed line represents unstable solution. 
nonmonotone solutions by reflecting and periodically extending the monotone ones at the end points.

According to Theorem 1.1, the shadow system (1.1) admits nonconstant monotone solutions for any small $\epsilon>0$. Therefore, from the view point of singular perturbations, we may expect that (1.2) also admits nontrivial solutions when $\chi$ and $d_{1}$ are sufficiently large, and although rigorous mathematical analysis is quite technical and is out of our scope, we perform extensive numerical simulations to verify this observation in Section 4.

\section{Nonconstant positive solutions to the shadow system}

To see how bifurcation analysis is performed in [1] and to introduce necessary notations and settings for our global analysis, we rewrite (1.1) into the abstract form

$$
\mathcal{F}(v, \lambda, \epsilon)=0, \quad(v, \lambda, \epsilon) \in \mathcal{X} \times \mathbb{R}^{+} \times \mathbb{R}^{+},
$$

where

$$
\mathcal{F}(v, \lambda, \epsilon)=\left(\begin{array}{c}
\epsilon v^{\prime \prime}+\left(a_{2}-b_{2} \lambda e^{-r \Phi(v)}-c_{2} v\right) v \\
\int_{0}^{L}\left(a_{1}-c_{1} v\right) e^{-r \Phi(v)} d x-b_{1} \lambda \int_{0}^{L} e^{-2 r \Phi(v)} d x
\end{array}\right)
$$

and $\mathcal{X}$ is the Hilbert space given by (1.7). We remark that any nonnegative solution $v$ of (1.1) in $\mathcal{X}$ is a classical solution thanks to the standard elliptic embeddings.

We first collect the following facts in [1] on the operator $\mathcal{F}$ before using the bifurcation theory.

Lemma 2.1 [1] The operator $\mathcal{F}(v, \lambda, \epsilon)$ defined in (2.1) satisfies the following properties:

(1) $\mathcal{F}(\bar{v}, \bar{\lambda}, \epsilon)=0$ for any $\epsilon \in \mathbb{R}^{+}$;

(2) $\mathcal{F}: \mathcal{X} \times \mathbb{R}^{+} \times \mathbb{R}^{+} \rightarrow \mathcal{Y} \times \mathcal{Y}$ is analytic, where $\mathcal{Y}=L^{2}(0, L)$;

(3) for any fixed $\left(v_{0}, \lambda_{0}\right) \in \mathcal{X} \times \mathbb{R}^{+}$, the Fréchet derivative of $\mathcal{F}$ is given by

$$
\begin{aligned}
& D_{(v, \lambda)} \mathcal{F}\left(v_{0}, \lambda_{0}, \epsilon\right)(v, \lambda) \\
& \quad=\left(\begin{array}{c}
\epsilon v^{\prime \prime}+\left(a_{2}-2 c_{2} v_{0}-b_{2} e^{-r \Phi\left(v_{0}\right)}\left(\lambda_{0}-\lambda_{0} v_{0} r \Phi^{\prime}\left(v_{0}\right)\right)\right) v-b_{2} e^{-r \Phi\left(v_{0}\right)} v_{0} \lambda \\
\int_{0}^{L}\left(b_{1} \lambda_{0} r \Phi^{\prime}\left(v_{0}\right) e^{-2 r \Phi\left(v_{0}\right)}-c_{1} e^{-r \Phi\left(v_{0}\right)}\right) v-b_{1} e^{-2 r \Phi\left(v_{0}\right)} \lambda d x
\end{array}\right) ;
\end{aligned}
$$

(4) $D_{(v, \lambda)} \mathcal{F}\left(v_{0}, \lambda_{0}, \epsilon\right): \mathcal{X} \times \mathbb{R}^{+} \rightarrow \mathcal{Y} \times \mathbb{R}$ is a Fredholm operator with zero index.

Besides these facts, we can further show that the dimension of the null space of $D_{(v, \lambda)} \mathcal{F}(\bar{v}, \bar{\lambda}, \epsilon)$ is 1 and the following necessary condition on the null space of operator (2.2) holds:

$$
\mathcal{N}\left(D_{(v, \lambda)} \mathcal{F}(\bar{v}, \bar{\lambda}, \epsilon)\right) \neq\{0\}
$$

and, in particular,

$$
\mathcal{N}\left(D_{(v, \lambda)} \mathcal{F}(\bar{v}, \bar{\lambda}, \epsilon)\right)=\operatorname{span}\{\cos (k \pi x / L), 0\}, \quad k \in \mathbb{N}^{+} .
$$


Moreover, we can verify the following transversality condition:

$$
\left.\frac{d}{d \epsilon}\left(D_{(v, \lambda)} \mathcal{F}(\bar{v}, \bar{\lambda}, \epsilon)\right)\left(v_{n}, \lambda_{n}\right)\right|_{\epsilon=\epsilon_{n}} \notin \mathcal{R}\left(D_{(v, \lambda)} \mathcal{F}\left(\bar{v}, \bar{\lambda}, \epsilon_{n}\right)\right)
$$

and therefore the existence of nonconstant bifurcating solutions follows from the classical Crandall-Rabinowitz bifurcation theory [14].

Remark 2 We want to point out that $\left(0, \frac{a_{1}}{b_{1}}\right)$ is the other constant solution to (1.1). However, it is easy to verify that Crandall-Rabinowitz bifurcation does not occur at this trivial solution. In fact, putting $\left(v_{0}, \lambda_{0}\right)=\left(0, \frac{a_{1}}{b_{1}}\right)$ in $(2.2)$, we see that the necessary condition (2.4) becomes

$$
\epsilon v^{\prime \prime}+\left(a_{2}-\frac{a_{1} b_{2}}{b_{1}}\right) v=0, \quad v^{\prime}(0)=v^{\prime}(L)=0,
$$

which has only the trivial solution, and hence the null-space $\mathcal{N}\left(D_{(v, \lambda)} \mathcal{F}\left(0, \frac{a_{1}}{b_{1}}, \epsilon\right)\right)$ must be trivial.

\section{Global bifurcation analysis}

We now proceed to extend the local bifurcation curves obtained in Theorem 1.1 by the global bifurcation theory of Rabinowitz [16] and its developed version in [17]. In particular, we will only study the first bifurcation branch $\Gamma_{1}$ since all the remaining (local) branches are unstable.

Denote the solution set of (1.1) by

$$
\mathcal{S}=\left\{(v, \lambda, \epsilon) \in \mathcal{X} \times \mathbb{R} \times \mathbb{R}^{+} \mid \mathcal{F}(v, \lambda, \epsilon)=0,(v, \lambda) \neq(\bar{v}, \bar{\lambda})\right\}
$$

and let $\mathcal{C}$ be the maximal connected subset of $\overline{\mathcal{S}}$ that contains $\left(\bar{v}, \bar{\lambda}, \epsilon_{1}\right)$. Then $\mathcal{C}$ is a closed set, and it contains $\left(v_{1}(x, s), \lambda_{1}(s), \epsilon_{1}(s)\right), s \in(-\delta, \delta)$. We show in the following lemma that all elements on $\mathcal{C}$ are solutions to (1.1) staying positive in this set.

Lemma 3.1 Assume that (1.4) holds. Then, for each $(v, \lambda, \epsilon) \in \mathcal{C}, v(x)>0$ on $[0, L], \lambda>0$, and $(\nu, \lambda)$ is a solution of $(1.1)$.

Proof We introduce the following connected set:

$$
\mathcal{P}_{0}=\{(v, \lambda) \in \mathcal{C} \mid v(x)>0, x \in[0, L], \lambda>0\} .
$$

First, we observe that $\mathcal{P}_{0}$ is a subset of $\mathcal{C}$, and it is nonempty since at least the part of $\mathcal{C}$ near $\left(\bar{v}, \bar{\lambda}, \epsilon_{1}\right)$ is contained in $\mathcal{P}_{0}$. Therefore, in order to prove the positivity of $v$ and $\lambda$, we need to show that $\mathcal{P}_{0}=\mathcal{C}$. For this purpose, we prove that $\mathcal{P}_{0}$ is both open and closed in $\mathcal{C}$ by topology arguments, and if this is done, the connectedness of $\mathcal{S}^{+}$implies that $\mathcal{P}_{0}=\mathcal{S}^{+}$.

To prove that $\mathcal{P}_{0}$ is open in $\mathcal{C}$, we pick any $(\nu, \lambda, \epsilon) \in \mathcal{P}_{0}$ and assume that there exists a sequence $\left(v_{k}, \lambda_{k}, \epsilon_{k}\right)$ that converges to $(v, \lambda, \epsilon)$ in $\mathcal{X} \times \mathbb{R} \times \mathbb{R}$. Therefore $v_{k}$ converges to $v$ in $C^{2}([0, L])$, and hence $v_{k}(x)>0$ on $[0, L]$ for all large $k$ since $v(x)>0$ on $[0, L]$. Moreover, $\lambda_{k}, \epsilon_{k}>0$ since $\lambda, \epsilon>0$. 
To prove that $\mathcal{P}_{0}$ is closed in $\mathcal{C}$, we take $\left\{\left(v_{k}, \lambda_{k}, \epsilon_{k}\right)\right\} \in \mathcal{P}_{0}$ such that, for some $(v, \lambda, \epsilon) \in \mathcal{C}$, $\left(v_{k}, \lambda_{k}, \epsilon_{k}\right)$ converges to $(v, \lambda, \epsilon)$ in the norm of $\mathcal{X} \times \mathbb{R}^{+} \times \mathbb{R}^{+}$. Then we want to show that $(v, \lambda, \epsilon) \in \mathcal{P}_{0}$, that is, $v(x)>0$ on $[0, L]$, and $\lambda>0$. Obviously, $v(x) \geq 0$ and $\lambda \geq 0$, and we only need to show that $\lambda \neq 0$ and $v(x)>0$ for all $x \in[0, L]$. We argue by contradiction. First, suppose that $\lambda=0$. Then the $v(x)$-equation in (1.1) becomes

$$
\left\{\begin{array}{l}
\epsilon v^{\prime \prime}+\left(a_{2}-c_{2} v\right) v=0, \quad x \in(0, L), \\
v^{\prime}(0)=v^{\prime}(L)=0
\end{array}\right.
$$

and it is well known that (3.1) has only the trivial solution 0 and $\frac{a_{2}}{c_{2}}$; therefore, $v_{k}$ converges to either 0 or $\frac{a_{2}}{c_{2}}$ uniformly on $[0, L]$. If $v_{k}$ converges to 0 , then we can apply the Lebesgue dominated convergence theorem to the integral constraint in (1.1):

$$
0=\lim _{k \rightarrow \infty} \int_{0}^{L}\left(a_{1}-b_{1} \lambda_{k} e^{-r \Phi\left(v_{k}\right)}-c_{1} v_{k}\right) e^{-r \Phi\left(v_{k}\right)} d x=a_{1} e^{-r \Phi(0)}>0,
$$

which is impossible. If $v_{k}$ converges to $\frac{a_{2}}{c_{2}}$, then similarly we have

$$
0=\lim _{k \rightarrow \infty} \int_{0}^{L}\left(a_{1}-b_{1} \lambda_{\epsilon} e^{-r \Phi\left(v_{\epsilon}\right)}-c_{1} v_{\epsilon}\right) e^{-r \Phi\left(v_{\epsilon}\right)} d x=\left(a_{1}-c_{1} \frac{a_{2}}{c_{2}}\right) e^{-r \Phi\left(\frac{a_{2}}{c_{2}}\right)}
$$

which implies that $\frac{a_{1}}{a_{2}}=\frac{c_{1}}{c_{2}}$, a contradiction to (1.4). Therefore, $\lambda=0$ is impossible, and we must have that $\lambda>0$, as claimed.

We now prove that $v(x)>0$ on $[0, L]$. If not, then suppose that $v\left(x_{0}\right)=0$ for some $x_{0} \in$ $[0, L]$. Then we can apply the strong maximum principle and Hopf's lemma to (1.1) to show that $v \equiv 0$ for all $x \in[0, L]$ and therefore $\lambda=\frac{a_{1}}{b_{1}}$. However, this is impossible since it is easy to check that bifurcation does not occur at $\left(0, \frac{a_{1}}{b_{1}}\right)$. This is a contradiction, and we must have that $v(x)>0$ on $[0, L]$.

Remark 3 According to Lemma 3.1 (and the forthcoming discussion), we know that the global continuum $\mathcal{C}$ cannot intersect with the $\epsilon$-axis. However, we are not able to rule out the possibility that it intersects with the $V$-axis, that is, $\mathcal{X} \times \mathbb{R}^{+} \times\{0\}$. Details on the limiting structures are needed for this purpose. Our main results in Theorem 1.1 establish the existence of nonconstant solutions to (1.1) for any small $\epsilon$, and it is out of the scope of this paper to analyze their limiting profiles.

We proceed to show that $\mathcal{C}$ consists of two disjoint components and each component contains solution $v$ that is spatially monotone. Let $\mathcal{C}_{u}$ to be the component of $\mathcal{C} \backslash\left\{\left(v_{1}(s, x), \lambda_{1}(s), \epsilon_{1}(s)\right) \mid s \in(-\delta, 0)\right\}$ containing $\left\{\left(v_{1}(s, x), \lambda_{1}(s), \epsilon_{1}(s)\right) \mid s \in[0, \delta)\right\}$ and, correspondingly, $\mathcal{C}_{l}=\mathcal{C} \backslash\left\{\left(\nu_{1}(s, x), \lambda_{1}(s), \epsilon_{1}(s)\right) \mid s \in(0, \delta)\right\}$ containing $\left\{\left(\nu_{1}(s, x), \lambda_{1}(s), \epsilon_{1}(s)\right) \mid s \in\right.$ $(-\delta, 0]\}$. Then we can readily see that $\mathcal{C}=\mathcal{C}_{u} \cup \mathcal{C}_{l}$ and $\mathcal{C}_{u} \cap \mathcal{C}_{l}=\left\{\left(\bar{v}, \bar{\lambda}, \epsilon_{1}\right)\right\}$. We have the following results.

Lemma 3.2 $\mathcal{C}_{u} \backslash\left(\bar{v}, \bar{\lambda}, \epsilon_{1}\right)$ consists of $(v, \lambda, \epsilon)$ with $v^{\prime}(x)>0$ on $(0, L)$, and $\mathcal{C}_{l} \backslash\left(\bar{v}, \bar{\lambda}, \epsilon_{1}\right)$ consists of $(v, \lambda, \epsilon)$ with $v^{\prime}(x)<0$ on $(0, L)$ and $\lambda, \epsilon>0$. 
Proof Our proof is based on the same topology arguments as in the proof of Lemma 3.1. We introduce the following four subsets:

$$
\begin{aligned}
& \mathcal{C}_{u}^{0}:=\mathcal{C}_{u} \backslash\left\{\left(\bar{v}, \bar{\lambda}, \epsilon_{1}\right)\right\}, \\
& \mathcal{P}_{1}^{+}:=\left\{(v, \lambda, \epsilon) \in \mathcal{X} \times \mathbb{R}^{+} \times \mathbb{R}^{+} \mid v(x)>0, v^{\prime}(x)>0, x \in(0, L)\right\}, \\
& \mathcal{C}_{l}^{0}:=\mathcal{C}_{l} \backslash\left\{\left(\bar{v}, \bar{\lambda}, \epsilon_{1}\right)\right\}, \\
& \mathcal{P}_{1}^{-}:=\left\{(v, \lambda, \epsilon) \in \mathcal{X} \times \mathbb{R}^{+} \times \mathbb{R}^{+} \mid v(x)>0, v^{\prime}(x)<0, x \in(0, L)\right\}
\end{aligned}
$$

Then Lemma 3.2 holds if we can prove that

$$
\mathcal{C}_{u}^{0} \subset \mathcal{P}_{1}^{+}, \quad \mathcal{C}_{l}^{0} \subset \mathcal{P}_{1}^{-}
$$

We only prove the first part, and the second one can be treated in the same way. We note that $\mathcal{C}_{u}^{0}$ is nonempty since any solution $(\nu, \lambda, \epsilon)$ of $(1.1)$ near $\left(\bar{v}, \bar{\lambda}, \epsilon_{1}\right)$ is in the set $\mathcal{C}_{u}^{0}$ thanks to (1.4). Since $\mathcal{C}_{u}^{0}$ is a connected subset of $\mathcal{X} \times \mathbb{R}^{+} \times \mathbb{R}^{+}$, we only need to show that $\mathcal{C}_{u}^{0} \cap \mathcal{P}_{1}^{+}$ is both open and closed with respect to the topology of $\mathcal{C}_{u}^{0}$, and we divide our proof into two parts.

To prove the openness, we assume that $(\tilde{v}, \tilde{\lambda}, \tilde{\epsilon}) \in \mathcal{C}_{u}^{0} \cap \mathcal{P}_{1}^{+}$and there exists a sequence $\left\{\left(\tilde{v}_{k}, \tilde{\lambda}_{k}, \tilde{\epsilon}_{k}\right)\right\}_{k=1}^{\infty}$ in $\mathcal{C}_{u}^{0}$ that converges to $(\tilde{v}, \tilde{\lambda}, \tilde{\epsilon})$ in the norm of $\mathcal{X} \times \mathbb{R}^{+} \times \mathbb{R}^{+}$. We want to show that, for all large $k,\left(\tilde{v}_{k}, \tilde{\lambda}_{k}, \tilde{\epsilon}_{k}\right) \in \mathcal{P}_{1}^{+}$, that is,

$$
\tilde{v}_{k}^{\prime}>0, \quad x \in(0, L), \quad \tilde{\lambda}_{k}>0, \quad \tilde{\epsilon}_{k}>0 .
$$

First, it is easy to see that $\tilde{\lambda}_{k}>0$ and $\tilde{\epsilon}_{k}>0$ since both have positive limits $\tilde{\lambda}>0$ and $\tilde{\epsilon}>0$. On the other hand, we conclude from $\tilde{v}_{k} \rightarrow \tilde{v}$ in $\mathcal{X}$ and from the elliptic regularity theory that $\tilde{v}_{k} \rightarrow \tilde{v}$ in $C^{2}([0, L])$. Differentiating the first equation of (1.1) with respect to $x$, we have

$$
\left\{\begin{array}{l}
\epsilon\left(\tilde{v}^{\prime}\right)^{\prime \prime}+\left(a_{2}-b_{2} \lambda_{\epsilon} e^{-r \Phi(\tilde{v})}+b_{2} \lambda_{\epsilon} e^{-r \Phi(\tilde{v})} \Phi^{\prime}(\tilde{v})-2 c_{2} \tilde{v}\right) \tilde{v}^{\prime}=0, \quad x \in(0, L), \\
\tilde{v}^{\prime}(0)=\tilde{v}^{\prime}(L)=0 .
\end{array}\right.
$$

By Hopf's lemma from the fact $\tilde{v}^{\prime}(x)>0$ we have that $\tilde{v}^{\prime \prime}(L)>0>\tilde{v}^{\prime \prime}(0)$, and then this second-order nondegeneracy implies that $\tilde{v}_{k}^{\prime}(x)>0$ in $(0, L)$. If not, suppose that there exists $x_{k} \in[0, L]$ such that $\tilde{v}_{k}^{\prime}\left(x_{k}\right)=0$. Then we have that $x_{k} \rightarrow x_{\infty} \in[0, L]$ as $k \rightarrow \infty$. We divide our discussions into the following two cases.

Case 1. $x_{\infty}=0, L$. We only consider $x_{\infty}=0$ since the same arguments can be applied for $x_{\infty}=L$. In this case,

$$
0>\tilde{v}^{\prime \prime}(0)=\lim _{k \rightarrow \infty} \frac{\tilde{v}^{\prime}\left(x_{k}\right)-\tilde{v}^{\prime}(0)}{x_{k}}=0,
$$

a contradiction.

Case 2. $x_{\infty} \in(0, L)$. This is also impossible by the continuity of $\tilde{v}^{\prime}(x)$. Therefore, we have that $\tilde{v}^{\prime}(x)>0$ in $(0, l)$ for $k$ large, and hence the openness is proved. 
To prove that $\mathcal{C}_{u}^{0} \cap \mathcal{P}_{1}^{+}$is closed in $\mathcal{C}_{u}^{0}$, we take a sequence $\left(\tilde{v}_{k}, \tilde{\lambda}_{k}, \tilde{\epsilon}_{k}\right) \in \mathcal{C}_{u}^{0} \cap \mathcal{P}_{1}^{+}$and assume that there exists $(\tilde{v}, \tilde{\lambda}, \tilde{\epsilon})$ in $\mathcal{C}_{u}^{0}$ such that $\left(\tilde{v}_{k}, \tilde{\lambda}_{k}, \tilde{\epsilon}_{k}\right) \rightarrow(\tilde{v}, \tilde{\lambda}, \tilde{\epsilon})$ in the topology of $\mathcal{X} \times \mathbb{R}^{+} \times \mathbb{R}^{+}$. We want to show that

$$
\tilde{v}^{\prime}(x)>0, \quad x \in(0, L), \quad \text { and } \quad \tilde{\lambda}>0 .
$$

By the same argument as before it can be easily proved that $\tilde{\lambda}>0$. We now need to show that $\tilde{v}^{\prime}(x)>0$. Again, we have from the elliptic regularity that $\tilde{v}_{k} \rightarrow \tilde{v}$ in $C^{2}([0, L])$, and therefore $\tilde{v}^{\prime}(x) \geq 0, x \in(0, L)$. Applying the strong maximum principle and Hopf's lemma to (3.2), we have that either $\tilde{v}^{\prime}>0$ or $\tilde{v}^{\prime} \equiv 0$ on $(0, L)$. In the latter case, we must have $(\tilde{v}, \tilde{\lambda}) \equiv(\bar{v}, \bar{\lambda})$, and this contradicts to the definition of $\mathcal{C}_{u}^{0}$. Thus, we have shown that $\tilde{v}^{\prime}>0$ on $(0, L)$, and this proves the closedness. The proof of Lemma 3.2 is complete.

\subsection{Global extension of the first bifurcation branch}

Finally, we study the extension of the local bifurcation branch $\Gamma_{1}(s)$, and we present a proof of Theorem 1.1.

Proof of Theorem 1.1 According to Theorem 4.4 in [17], $\mathcal{C}_{u}$ satisfies one of the following three alternatives: (A1) it is not compact in $\mathcal{X} \times \mathbb{R}^{+} \times \mathbb{R}^{+}$; (A2) it contains a point $\left(\bar{v}, \bar{\lambda}, \epsilon_{*}\right)$ with $\epsilon_{*} \neq \epsilon_{1} ;$ (A3) it contains a point $(\bar{v}+\hat{v}, \bar{\lambda}+\hat{\lambda}, \epsilon)$, where $0 \neq(\hat{v}, \hat{\lambda}) \in \mathcal{Z}$, and $\mathcal{Z}$ is a closed complement of $\mathcal{N}\left(D_{(v, \lambda)} \mathcal{F}\left(\bar{v}, \bar{\lambda}, \epsilon_{1}\right)\right)=\operatorname{span}\left\{\left(\cos \frac{\pi x}{L}, 0\right)\right\}$. We first claim that only alternative (A1) can occur.

If (A2) occurs, then $\epsilon_{*}$ must be one of the bifurcation values $\epsilon_{k}, k \geq 2$; therefore, $v(x)=\bar{v}+$ $s \cos \frac{k \pi x}{L}+o(s), s \in(-\delta, \delta)$, according to (1.4). However, this contradicts to the monotonicity of $v(x)$, and therefore (A2) is impossible.

If (A3) occurs, then we can choose the complement to be

$$
\mathcal{Z}=\left\{(v, \lambda) \in \mathcal{X} \times \mathbb{R}^{+} \mid \int_{0}^{L} v(x) \cos \frac{\pi x}{L} d x=0\right\},
$$

and then, for any $(v, \lambda) \in \mathcal{Z}$, by integration by parts we have

$$
0=\int_{0}^{L} v(x) \cos \frac{\pi x}{L} d x=-\frac{L}{\pi} \int_{0}^{L} v^{\prime}(x) \sin \frac{\pi x}{L} d x<0,
$$

a contradiction. Therefore, only alternative (A1) occurs, and $\mathcal{C}_{u}$ is not compact in $\mathcal{X} \times$ $\mathbb{R}^{+} \times \mathbb{R}^{+}$.

Now we study the behavior of $\mathcal{C}_{u}$, and that of $\mathcal{C}_{l}$ can be investigated in the same way. First, we claim that the projection of $\mathcal{C}_{u}$ onto the $\epsilon$-axis does not contain an interval of the form $\left(\epsilon_{0}, \infty\right)$ for any $\epsilon_{0}>0$ and it is sufficient to show that there exist a positive constant $\bar{\epsilon}_{0}$ such that (1.1) has only constant positive solution $(\bar{v}, \bar{\lambda})$ if $\epsilon \in\left(\bar{\epsilon}_{0}, \infty\right)$. To prove the claim, we decompose the solution $v(x)$ of $(1.1)$ as

$$
v=\bar{v}+w,
$$

where $\bar{v}=\frac{1}{L} \int_{0}^{L} v d x$ and $\int_{0}^{L} w d x=0$. Then we readily see that $w$ satisfies

$$
\left\{\begin{array}{l}
\epsilon w^{\prime \prime}+\left(a_{2}-b_{2} \lambda e^{-r \Phi(\bar{v}+w)}-c_{2} \bar{v}-c_{2} w\right)(\bar{v}+w)=0, \quad x \in(0, L), \\
\int_{0}^{L} w d x=0, \quad w^{\prime}(0)=w^{\prime}(L)=0 .
\end{array}\right.
$$


Multiplying both hand sides of (3.3) by $w$ and then integrating over $(0, L)$, we have that

$$
\epsilon \int_{0}^{L}\left(w^{\prime}\right)^{2} d x=\left(a_{2}-2 c_{2} \bar{v}\right) \int_{0}^{L} w^{2} d x-b_{2} \lambda \int_{0}^{L} e^{-r \Phi((\bar{v}+w))}(\bar{v}+w) d x-c_{2} \int_{0}^{L} w^{3} d x .
$$

On the other hand, it is easy to have from the maximum principle that both $\left\|v_{\epsilon}(x)\right\|_{L^{\infty}(0, L)}$ and $\lambda_{\epsilon}$ are uniformly bounded in $\epsilon$. Then we have from the last equality that

$$
\epsilon \int_{0}^{L}\left(w^{\prime}\right)^{2} d x \leq C_{0} \int_{0}^{L} w^{2} d x
$$

where $C_{0}$ is a positive constant dependent on $a_{2}, c_{2}$, and $\bar{v}$. We reach a contradiction if $\epsilon>$ $\frac{C_{0}}{(\pi / L)^{2}}$ unless $w \equiv 0$. Therefore, we must have that $v \equiv \bar{v}$; however, this gives a contradiction by the same arguments as in case (A2). Therefore, the claim is proved.

Now we proceed to show that the projection $\mathcal{C}_{u}$ onto the $\epsilon$-axis is of the form $(0, \bar{\epsilon}]$ for some $\bar{\epsilon} \geq \epsilon_{1}$. We argue by contradiction and assume that there exists $\underline{\epsilon}>0$ such that $(\underline{\epsilon}, \bar{\epsilon})$ is contained in this projection, but this projection does not contain any $\epsilon<\underline{\epsilon}$. Then we have from the uniform boundedness of $\left\|v_{\epsilon}(x)\right\|_{\infty}$ and Sobolev embedding that, for each $\epsilon>0,\left\|v_{\epsilon}\right\|_{C^{3}([0, L])} \leq C$ for all $\left(v_{\epsilon}, \lambda_{\epsilon}, \epsilon\right) \in \mathcal{C}_{u}$. However, this implies that $\mathcal{C}_{u}$ is compact in $\mathcal{X} \times \mathbb{R}^{+} \times \mathbb{R}^{+}$, which is a contradiction to alternative (A1). Therefore, $\mathcal{C}_{u}$ extends to infinity vertically in $\mathcal{X} \times \mathbb{R}^{+} \times \mathbb{R}^{+}$. This finishes the proof of Theorem 1.1.

We have from Theorem 1.1 that there exist positive and monotone solutions $v_{\epsilon}\left(\lambda_{\epsilon}, x\right)$ to (1.1) for all $\epsilon \in\left(0, \epsilon_{1}\right)$. If $v(x)$ is an increasing solution to (1.1), then $v(L-x)$ is a decreasing solution. Then we can construct infinitely many nonmonotone-solutions of (1.1) by reflecting and periodically extending $v(x)$ and $v(L-x)$ at $x=0, \pm L, \pm 2 L, \ldots$

\section{Numerical simulations}

We proceed to investigate (1.1) and (1.2) by numerical studies. Our simulation illustrates and supports our theoretical finding in the previous sections, that is, (1.2) admits nontrivial positive constant steady states when $\chi$ and $d_{1}$ are large and $d_{2}$ is small. Moreover, system (1.2) is able to model the well-observed phenomenon of segregation through competition.

According to [1], in limit of large $\chi$ and $d_{1}$, positive solutions $(u, v)$ of $(1.2)$ over $\Omega=(0, L)$ can be approximated by those of the shadow system (1.1). Moreover, thanks to Theorem 1.1, the boundary value problem (1.1) admits nonconstant solutions for any small $\epsilon>0$; therefore, we expect the emergence of nontrivial patterns in (1.2) for $\chi$ and $d_{1}$ being large and $d_{2}$ being sufficiently small. We choose the sensitivity function to be $\phi(v) \equiv 1$ without losing much generality of our numerical studies. In the remaining part of this section, we shall numerically study the following coupled time-dependent system:

$$
\begin{cases}u_{t}=\nabla \cdot\left(d_{1} \nabla u+\chi u \nabla v\right)+\left(a_{1}-b_{1} u-c_{1} v\right) u=0, & x \in \Omega, t>0, \\ v_{t}=d_{2} \Delta v+\left(a_{2}-b_{2} u-c_{2} v\right) v=0, & x \in \Omega, t>0, \\ u_{x}(x, t)=v_{x}(x, t)=0, & x=\partial \Omega, t>0, \\ u(x, 0)=u_{0}, \quad v(x, 0)=v_{0}(x), & x \in \Omega .\end{cases}
$$

Then their stationary solutions correspond to those of (1.2). Choosing the system parameters $a_{1}=a_{2}=b_{2}=c_{1}=1$ and $b_{1}=c_{2}=2$, it is easy to find that $(\bar{u}, \bar{v})=\left(\frac{1}{3}, \frac{1}{3}\right)$ is a 
trivial solution. Moreover, we choose $\chi=500, d_{1}$, and the initial data $\left(u_{0}, v_{0}\right)=(\bar{u}, \bar{v})+$ $(0.001,0.001) \cos \pi x$ in all the simulations (except the initial data in Figure 9). It is our goal to examine the effect of $d_{2}$ (or, equivalently, of $\epsilon$ in (1.1)) and the domain size on the formation of nontrivial patterns in (1.2). Our numerics demonstrate the self-organized spatial temporal dynamics of these systems.

\subsection{Simulations over one-dimensional domains}

In Figure 2, we plot the spatial-temporal solutions to illustrate the formation of single boundary spike to $u$ and boundary layer to $v$. The boundary spike and layer here correspond to the monotone steady state obtained in Theorem 1.1. Moreover, the numerical
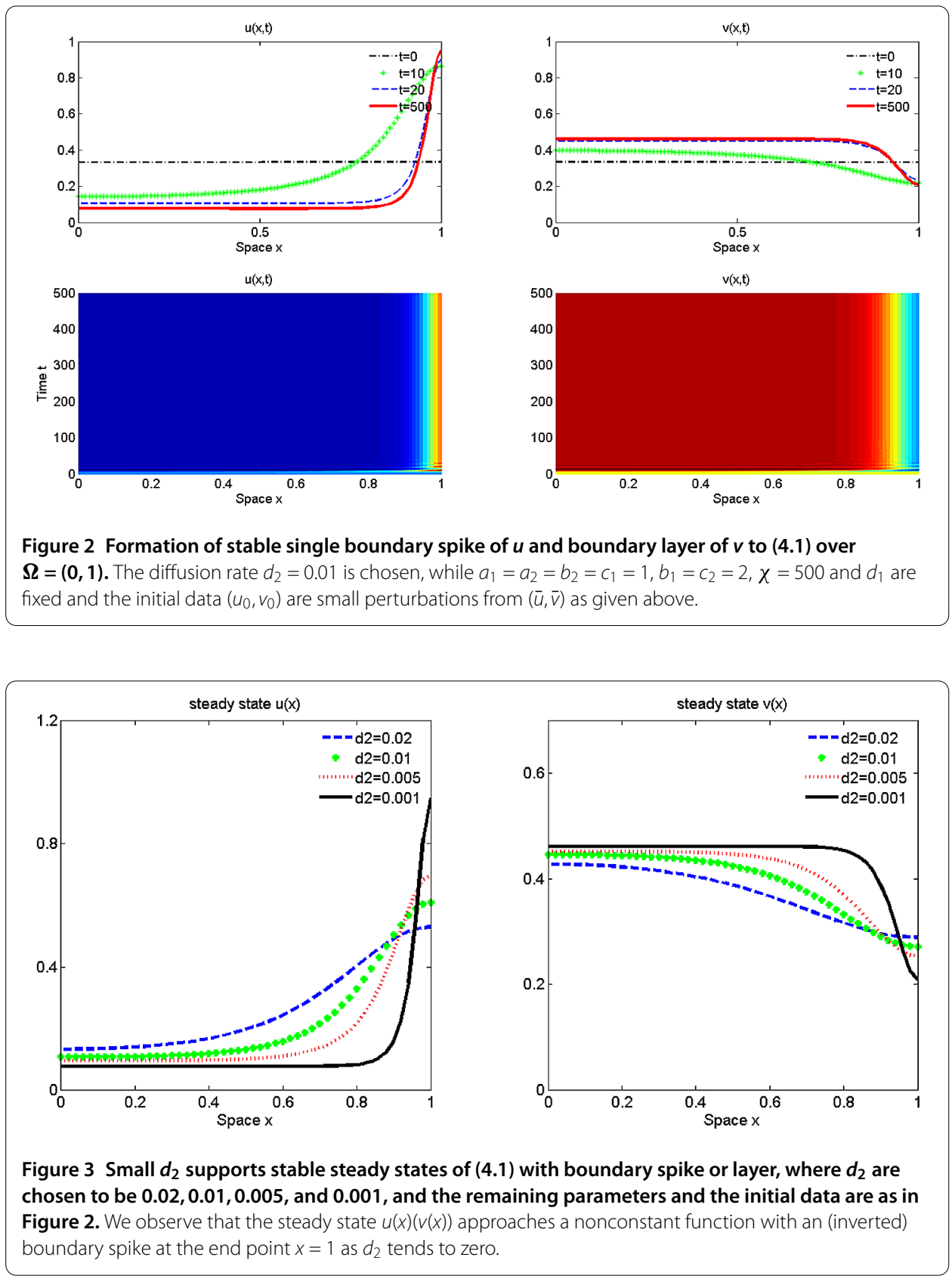
simulations suggest that the spiky solutions are stable. The rigorous proof of the stability is out of scope of this paper.

According to Theorem 1.1, (1.1) admits nonmonotone positive solutions for any small $\epsilon>0$; therefore, it is natural to expect that the same holds for (4.1) when both $\chi$ and $d_{1}$ are large. On the other hand, we know from our proof of Theorem 1.1 that the large $d_{2}$ inhibits the existence of nonconstant positive solutions. Therefore, we present Figure 3 to investigate the effect of small $d_{2}$ on the nonconstant steady states to (4.1). As $d_{2}$ tends to zero, the boundary spike shifts to the boundary with its magnitude increases. However, rigourous analysis of these spiky solutions is quite challenging and is out of the scope of this paper.
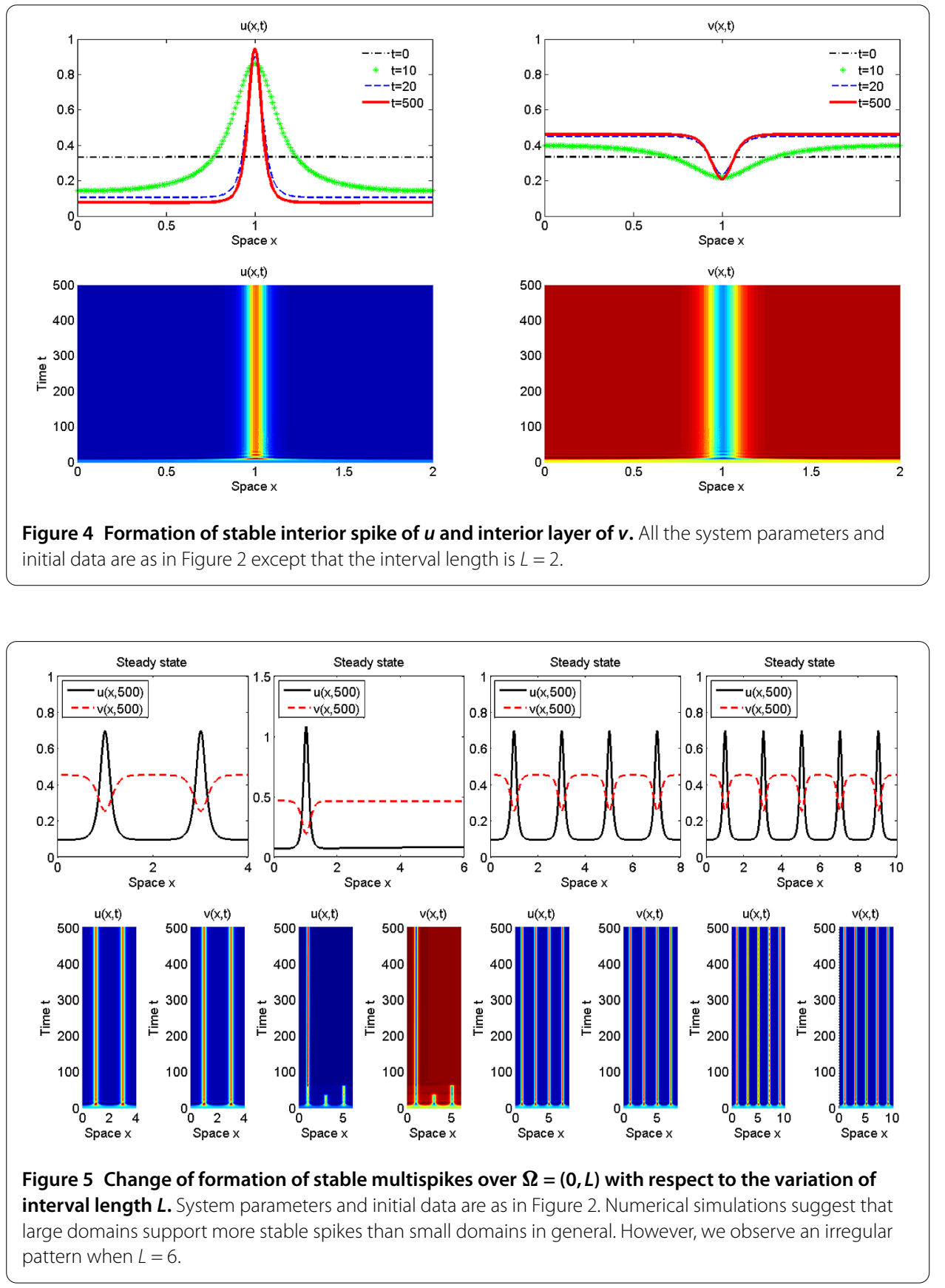
Thanks to the Neumann boundary conditions, we can construct single- and multiinterior spikes for (4.1) by reflecting and periodically extending the single boundary spike at the end points. This is numerically demonstrated in Figure 4.

In Figure 5, we continue to examine the effect of large interval on the formation of multispikes in (4.1). We observe the reflection and periodic extension of spikes over interval with $L=2$ to those $L=4$ and $L=8$. However, we may expect steady states with three spikes when $L=6$, admitting a single interior instead. We are motivated to study the dynamics of such an irregular pattern observed in Figure 5 when $L=6$. To do so, we select $L=16$ and plot in Figure 6 the profile of $u(x, t)$ for specifically chosen times. We observe $u(x, t)$ develops with multispikes which are meta-stable, the stability of which is destroyed at time
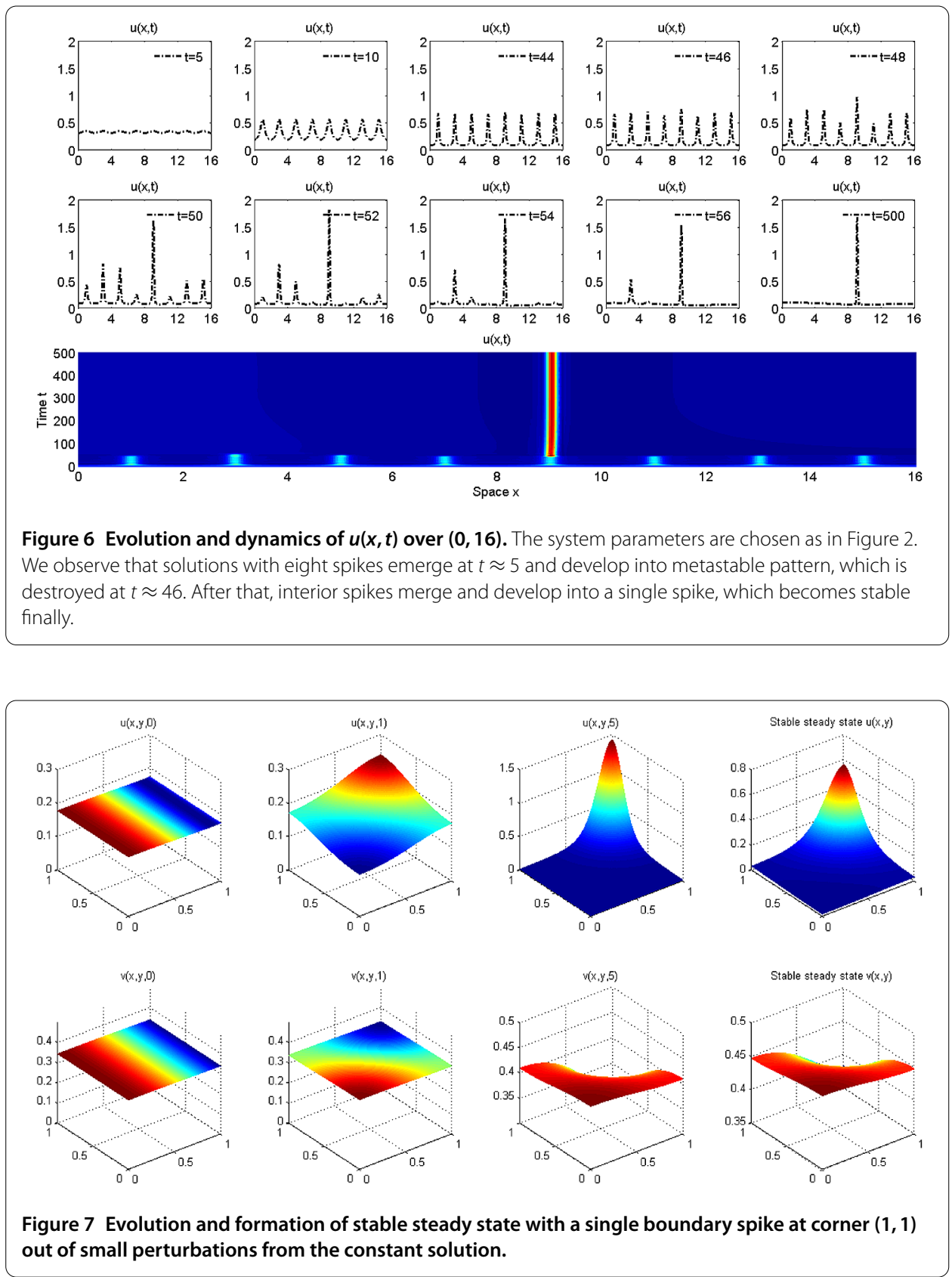

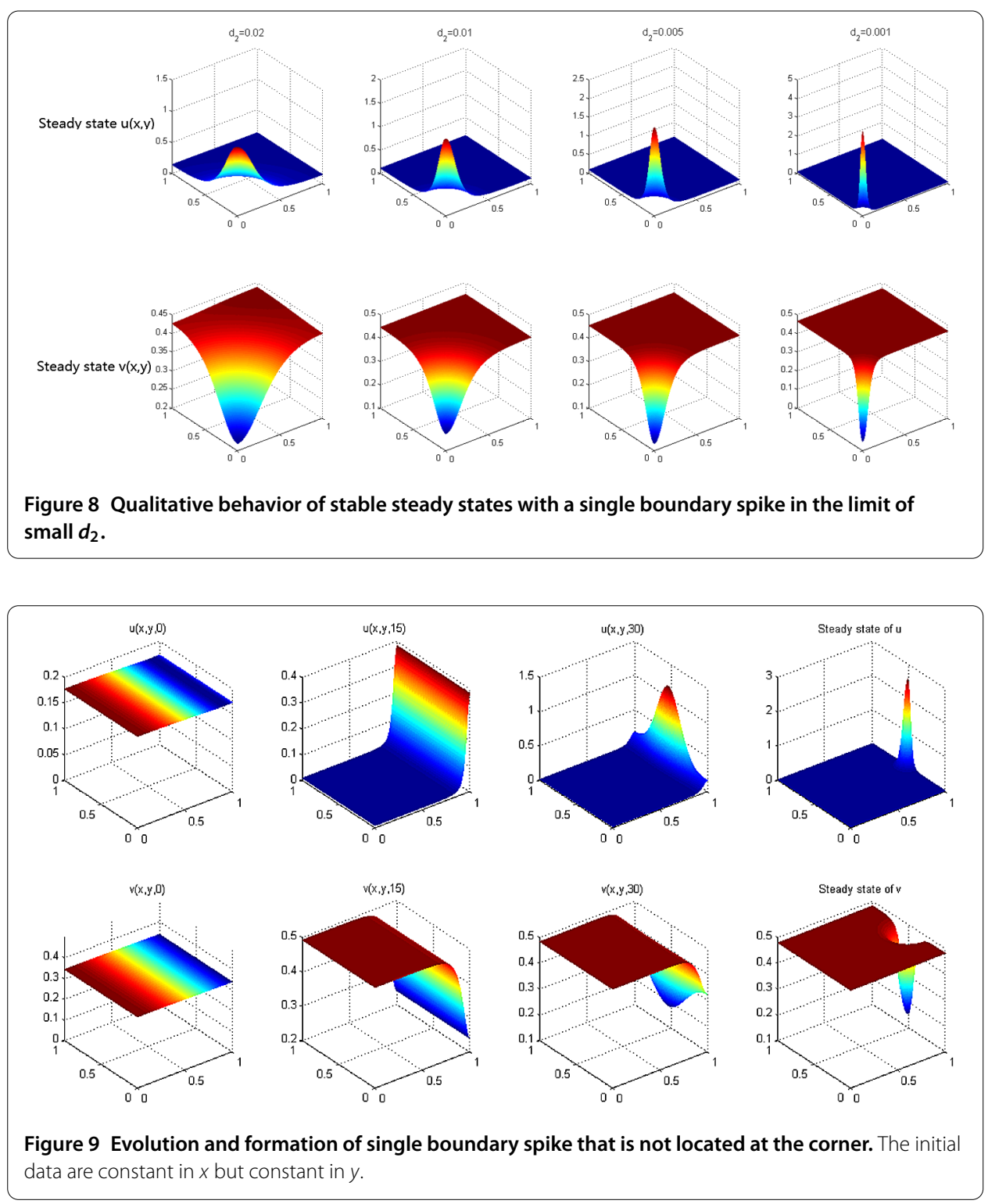

$t \approx 46$. Then we observe the merging and disappearing of the interior spikes, and finally $u(x, t)$ develops into a single interior spike afterwards.

\subsection{Simulations over two-dimensional domains}

We now study (4.1) over the square $\Omega=(0, L) \times(0, L)$. Similarly as in the $1 \mathrm{D}$ case, we shall examine the effect of $d_{2}$ and the domain size over the formation and evolution of nontrivial patterns, whereas the remaining system parameters and initial data are chosen as in Figure 2. For example, in Figure 7, we plot the formation and evolution of stable boundary spike out of small perturbations from the constant solution $(\bar{u}, \bar{v})$. Similarly as in Figure 3, we show in Figure 8 that when $d_{2}$ becomes small, the boundary spike shifts to the corner and its magnitude increases. It seems necessary to point out, a stable single boundary spike does not have to stay at the corner as we observe one counter-example in Figure 9. Finally, we include Figure 10 to demonstrate that large domains support stable steady states with more spikes than small domains. Mathematical analysis of the spatial- 


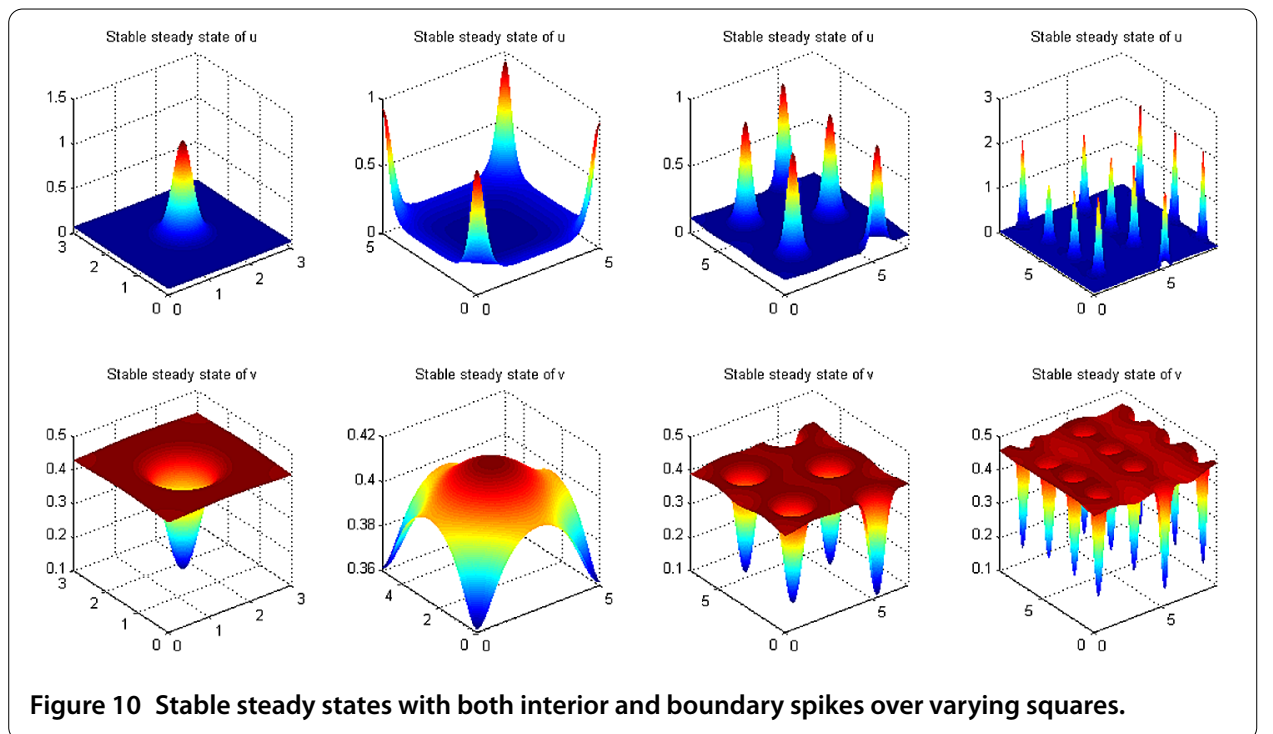

temporal of these spikes is an interesting but also quite demanding question that we can study in the future.

\section{Competing interests}

The authors declare that they have no competing interests.

\section{Authors' contributions}

Both authors read and approved the final manuscript and contributed equally to the writing of this paper. Numerical simulations are mainly due to $Y Z$, and the analysis is mainly due to $L X$.

\section{Author details}

${ }^{1}$ School of Securities and Futures, Southwestern University of Finance and Economics, Chengdu, 611130, China. ${ }^{2}$ College of Mathematics and Statistics, Guangdong University of Finance and Economics, Guangzhou, Guangdong 510320, China.

${ }^{3}$ College of Mathematics and Statistics, Shenzhen University, Shenzhen, Guangdong 518060, China.

\section{Acknowledgements}

YZ supported by Department of Education, Sichuan (15ZB0473). LX supported by Guangdong Natural Science Foundation (2015A030313623) and Guangdong Training Program for Young College Teachers (YQ2015077).

Received: 5 October 2016 Accepted: 6 January 2017 Published online: 25 January 2017

\section{References}

1. Wang, Q, Gai, C, Yan, J: Qualitative analysis of a Lotka-Volterra competition system with advection. Discrete Contin. Dyn. Syst. 35, 1239-1284 (2015)

2. De Mottoni, P, Rothe, F: Convergence to homogeneous equilibrium state for generalized Volterra-Lotka systems with diffusion. SIAM J. Appl. Math. 37, 648-663 (1979)

3. Kishimoto, K, Weinberger, $\mathrm{H}$ : The spatial homogeneity of stable equilibria of some reaction-diffusion systems in convex domains. J. Differ. Equ. 58, 15-21 (1985)

4. Matano, H, Mimura, M: Pattern formation in competition-diffusion systems in nonconvex domains. Publ. Res. Inst. Math. Sci. 19, 1049-1079 (1983)

5. Mimura, M, Ei, S-I, Fang, Q: Effect of domain-shape on coexistence problems in a competition-diffusion system. J. Math. Biol. 29, 219-237 (1991)

6. Mimura, M, Kawasaki, K: Spatial segregation in competitive interaction-diffusion equations. J. Math. Biol. 9, 49-64 (1980)

7. Mimura, M, Nishiura, Y, Tesei, $A$, Tsujikawa, T: Coexistence problem for two competing species models with density-dependent diffusion. Hiroshima Math. J. 14, 425-449 (1984)

8. Shigesada, N, Kawasaki, K, Teramoto, E: Spatial segregation of interacting species. J. Theor. Biol. 79, 83-99 (1979)

9. Choi, YS, Lui, R, Yamada, Y: Existence of global solutions for the Shigesada-Kawasaki-Teramoto model with weak cross-diffusion. Discrete Contin. Dyn. Syst. 9, 1193-1200 (2003)

10. Kuto, K: Limiting structure of shrinking solutions to the stationary Shigesada-Kawasaki-Teramoto model with large cross-diffusion. SIAM J. Math. Anal. 47, 3993-4024 (2015)

11. Lou, Y, Ni, W-M, Yotsutani, S: On a limiting system in the Lotka-Volterra competition with cross-diffusion. Discrete Contin. Dyn. Syst. 10, 435-458 (2004)

12. Keller, EF, Segel, LA: Inition of slime mold aggregation view as an instability. Journal of Theoratical Biology 26 , 399-415 (1970) 
13. Kuto, K, Tsujikawa, T: Limiting structure of steady-states to the Lotka-Volterra competition model with large diffusion and advection. J. Differ. Equ. 258, 1801-1858 (2015)

14. Crandall, MG, Rabinowitz, PH: Bifurcation from simple eigenvalues. J. Funct. Anal. 8, $321-340$ (1971)

15. Ni, W-M, Polascik, P, Yanagida, E: Monotonicity of stable solutions in shadow systems. Transl. Am. Math. Soc. 353, 5057-5069 (2001)

16. Rabinowitz, PH: Some global results for nonlinear eigenvalue problems. J. Funct. Anal. 7, 487-513 (1971)

17. Shi, J, Wang, X: On global bifurcation for quasilinear elliptic systems on bounded domains. J. Differ. Equ. 246, 2788-2812 (2009)

Submit your manuscript to a SpringerOpen ${ }^{\circ}$ journal and benefit from:

- Convenient online submission

Rigorous peer review

- Immediate publication on acceptance

- Open access: articles freely available online

- High visibility within the field

- Retaining the copyright to your article 